\title{
A METHOD FOR MODELLING VARYING RUN-OFF EVOLUTIONS IN CLAIMS RESERVING
}

\author{
By R.J. VERRALL \\ Department of Actuarial Science and Statistics \\ City University \\ London
}

\begin{abstract}
This paper considers the application of state space modelling to the chain ladder linear model in order to allow the run-off parameters to vary with accident year. In the usual application of the chain ladder technique, the development factors are assumed to be the same for each accident year. This implies that the run-off shape does not alter with accident year. This paper shows how this assumption can be relaxed in order to allow a recursive smooth model to be applied, or for large changes in the shape of the run-off curve. It is possible for these changes to be modelled using external inputs, or for a multiprocess model to be used to detect changes in the run-off shape.
\end{abstract}

\section{KEYWORDS}

Chain ladder technique; Kalman filter; linear models; state space models.

\section{INTRODUCTION}

The claims reserving process is made up of two parts. The first part is the analysis of the data, and the fitting of suitable models. The second part consists of using the results of the modelling process to project future claims experience. This paper is concerned primarily with the model fitting procedure: the use of the models for forecasting will be discussed only briefly. In addition, attention is restricted to the development factors and no attempt is made to provide a comprehensive claims analysis procedure. Thus, this paper shows how to modify the chain ladder linear model when there are indications that the run-off pattern is changing. This change might be gradual or sudden: the value of the method presented here is that it allows the change to be incorporated into the reserving process.

In order to project future claims it is necessary to have as full an understanding of the pattern of claims experience as possible. The chain ladder technique, which is widely used, does not allow the run-off pattern to change from accident year to accident year. It is unlikely that evidence that the run-off pattern has changed will come to light when the chain ladder technique is used in its usual form. The purpose of this paper is to adapt the chain ladder technique to allow the development factors to evolve with accident year. 


\section{THE CHAIN LADDER LINEAR MODEL}

In order to apply the recursive smoothing methods, we first write the chain ladder technique as a linear model. The original reference for this is KREMER (1982) and a useful later reference is RENSHAw (1989). We will not give full details here as these can be found in the above papers and in VerRaLl (1989) and Verrall (1991a).

The chain ladder technique is based on the following model:

$$
E\left[C_{i j} \mid C_{i, j-1}, \ldots, C_{i 1}\right]=\lambda_{j} C_{i, j-1}
$$

where

$C_{i j}=$ cumulative claims in accident year $i$, development year $j$.

$\lambda_{j}=$ development factor for year $j$.

Define the incremental claims by $Z_{i j}$ where

$$
\begin{aligned}
& Z_{i j}=C_{i j}-C_{i, j-1} \quad j \geq 2 \\
& Z_{i 1}=C_{i 1}
\end{aligned}
$$

The logged incremental claims are denoted by $Y_{i j}$ where $Y_{i j}=\log \left(Z_{i j}\right)$. The chain ladder linear model is

$$
E\left(Y_{i j}\right)=\mu+\alpha_{i}+\beta_{j}
$$

with the constraints that $\alpha_{1}=\beta_{1}=0$.

Because the model has a row and column effect, the parameters are called the row parameters $\left(\alpha_{i}\right)$ and column parameters $\left(\beta_{j}\right)$. The following relationship between the column parameters was derived in VERRALL (1991b):

$$
\lambda_{j}=1+\frac{e^{\beta_{j}}}{\sum_{k=1}^{j-1} e^{\beta_{k}}}
$$

The chain ladder technique estimates the development factors by $\tilde{\lambda}_{j}$, where

$$
\tilde{\lambda}_{j}=\frac{\sum_{i=1}^{n-j+1} C_{i j}}{\sum_{i=1}^{n-j+1} C_{i, j-1}}
$$

(assuming we have a $n \times n$ run-off triangle).

This can be seen to be a weighted average of the estimate of the development factor for each row, the weights being the cumulative claims in development year $j-1$. The estimates from each row are

$$
\frac{C_{1 j}}{C_{1, j-1}}, \frac{C_{2 j}}{C_{2, j-1}}, \ldots, \frac{C_{n-j+1, j}}{C_{n-j+1, j-1}}
$$

and the weights are $C_{1, j-1}, C_{2, j-1}, \ldots, C_{n-j+1, j-1}$. 
This gives the estimate $\tilde{\lambda}_{j}$ in equation (4). The chain ladder technique is often criticised because it does not allow the run-off shape to evolve, as it imposes the same development factors on each row. An alternative model would be

$$
E\left[C_{i j} \mid C_{i, j-1}, \ldots, C_{i 1}\right]=\lambda_{i j} C_{i, j-1}
$$

This is obviously unreasonable since there would be far too many parameters. The model which will be studied in this paper lies between these two extremes. It will be assumed that the development parameters are similar from row to row, but not identical. The extension uses a state space model in a similar way to VERRALL (1989). However, that paper did not address the development factors in any detail, and the recursive relationship defined here has not been considered before. The estimates of the development factors in the chain ladder technique will be found from equation (3).

The next section describes the state space model which allows the development factors to vary from row to row.

\section{THE STATE SPACE MODEL}

This section contains a summary of the state space model which was derived in VerRall (1989) and shows how to extend it to allow the run-off pattern to evolve stochastically. The data which make up the claims run-off triangle can be regarded as a time series, and in year $t$ the data which are received are

$$
\left[\begin{array}{c}
Z_{1, t} \\
Z_{2, t-1} \\
\vdots \\
Z_{t, 1}
\end{array}\right]
$$

The chain ladder linear model, given by equation (2), can be written in matrix form for the data at time $t$ as

$$
\underline{Y}_{t}=F_{t} \underline{\theta}_{t}+\underline{e}_{t}
$$

where $F_{t}$ is the matrix which specifies the model and $\underline{\theta}_{t}$ is the parameter vector at time $t$.

VERRALL (1989) gives the model for the basic chain ladder technique, but it is necessary to extend it to separate the development parameters in each accident year. Thus, it is necessary to differentiate between $\beta_{1,2}$ and $\beta_{2,2}$, where $\beta_{1,2}$ and $\beta_{2,2}$ are the original column parameter $\beta_{2}$, but in rows 1 and 2 respectively. Unfortunately, it is hard (and not helpful) to define the general form of the model at time $t$, but we can see the way it can be done by considering times $t=2$ and 3 (say):

$$
\left[\begin{array}{l}
Y_{1,2} \\
Y_{2,1}
\end{array}\right]=\left[\begin{array}{lll}
1 & 0 & 1 \\
1 & 1 & 0
\end{array}\right]\left[\begin{array}{c}
\mu \\
\alpha_{2} \\
\beta_{1,2}
\end{array}\right]+\left[\begin{array}{c}
e_{1,2} \\
e_{2,1}
\end{array}\right]
$$


(8)

$$
\left[\begin{array}{l}
Y_{1,3} \\
Y_{2,2} \\
Y_{3,1}
\end{array}\right]=\left[\begin{array}{llllll}
1 & 0 & 0 & 0 & 0 & 1 \\
1 & 1 & 0 & 0 & 1 & 0 \\
1 & 0 & 1 & 0 & 0 & 0
\end{array}\right]\left[\begin{array}{c}
\mu \\
\alpha_{2} \\
\alpha_{3} \\
\beta_{1,2} \\
\beta_{2,2} \\
\beta_{1,3}
\end{array}\right]+\left[\begin{array}{l}
e_{1,3} \\
e_{2,1} \\
e_{3,1}
\end{array}\right]
$$

These equations, the observation equations, form one part of the state space model. The difference between this model and the standard chain ladder linear model is that the column parameters are not the same in each row. This can be seen by considering two observations in the same column, for example $Y_{2,3}$ and $Y_{3,3}$. The standard model is:

$$
\begin{aligned}
& Y_{2,3}=\mu+\alpha_{2}+\beta_{3}+e_{2.3} \\
& Y_{3,3}=\mu+\alpha_{3}+\beta_{3}+e_{3,3}
\end{aligned}
$$

and the new model is

$$
\begin{aligned}
& Y_{2,3}=\mu+\alpha_{2}+\beta_{2,3}+e_{2,3} \\
& Y_{3,3}=\mu+\alpha_{3}+\beta_{3,3}+e_{3,3}
\end{aligned}
$$

The state space model connects $\beta_{2,3}$ to $\beta_{3,3}$, but does not insist that $\beta_{3,3}=\beta_{2,3}$. The connection is made by the system equations which, in their most general form, are as follows

$$
\underline{\theta}_{t+1}=G_{t} \underline{\theta}_{t}+H_{t} \underline{u}_{t}+\underline{w}_{t} .
$$

It can be seen that the system equation relates the parameter vector at time $t+1$ to the parameter vector at time $t$. The matrix $G_{t}$ governs the exact form of this relationship. The vector $\underline{u}_{t}$ contains any new parameters at time $t$ which are not related to those at time $t-1$. In this case these will be the column parameters for the new column which is added to the triangle at time $t$. It is usual to use vague prior distributions for each input $\underline{u}_{l}$, reflecting the fact that there is unlikely to be information about the parameters before any relevant data are received. $\underline{w}_{t}$ is a zero-mean stochastic disturbance term.

The model is mostly defined by the form of the matrix $G_{t}$. In this paper, it is chosen so that the column parameters evolve in the following way:

\section{Column 2}

Row

2
3
4
$\vdots$

$$
\begin{array}{ll}
\beta_{2,2}=\beta_{1,2}+w_{2,2} & \beta_{2,3}=\beta_{1,3}+w_{2,3} \\
\beta_{3,2}=\beta_{2,2}+w_{3,2} & \beta_{3,3}=\beta_{2,3}+w_{3,3} \\
\beta_{4,2}=\beta_{3,2}+w_{4,2} &
\end{array}
$$

If the stochastic disturbance terms have zero variance, then the column parameters are identical in each row and the model reverts to the basic chain ladder linear model. The larger the variance, the more variation is allowed between the rows. These variances can be chosen by the user. They can be the same for each row and 
column, or can differ according to prior opinion on changes in the run-off pattern. For example, if there is evidence that the initial rows form a homogeneous group, but that there is then a change in the run-off pattern, larger variances terms can be included to allow this change to be reflected in the column parameters.

It has often been remarked in previous papers that the chain ladder technique is over-parameterised, due to using a separate parameter, $\alpha_{t}$, for each row. This criticism is of the opposite form to that made of the development factors. The use of a separate parameter for each row effect makes too little connection between the rows, while the use of identical development factors for each row makes too great a connection between the rows. The usual way to overcome the problem with the row parameters is to use a recursive model for these parameters. This is defined by

$$
\alpha_{t+1}=\alpha_{t}+v_{t}
$$

where $v_{t}$ is a zero-mean stochastic disturbance term.

Continuing with the illustration for the model at times $t=2$ and 3 , the system equation which relates the parameter vector at time $t=3$ to that at time $t=2$ is

$$
\left[\begin{array}{c}
\mu \\
\alpha_{2} \\
\alpha_{3} \\
\beta_{1,2} \\
\beta_{2,2} \\
\beta_{1,3}
\end{array}\right]=\left[\begin{array}{lll}
1 & 0 & 0 \\
0 & 1 & 0 \\
0 & 1 & 0 \\
1 & 0 & 0 \\
1 & 0 & 0 \\
0 & 0 & 0
\end{array}\right]\left[\begin{array}{c}
\mu \\
\alpha_{2} \\
\beta_{1,2}
\end{array}\right]+\left[\begin{array}{l}
0 \\
0 \\
0 \\
0 \\
0 \\
1
\end{array}\right] u_{3}+\left[\begin{array}{c}
0 \\
0 \\
v_{2} \\
0 \\
w_{2,2} \\
0
\end{array}\right]
$$

We have now defined a state space model which allows the run-off pattern to change from row to row. This model can be fitted using the Kalman Filter, as was described in VERRALL (1989). The next section contains a numerical illustration of this model.

\section{NUMERICAL ILLUSTRATION}

As a numerical illustration, the data which has been used previously by the author is again used in this paper. It should be emphasised that this is for illustration purposes: the whole range of claims data is varied enough that comprehensive examples covering all possibilities are not feasible. The data is taken from TAYLOR and AsHE (1983).

$\begin{array}{rrrrrrrrrr}357848 & 766940 & 610542 & 482940 & 527326 & 574398 & 146342 & 139950 & 227229 & 67948 \\ 352118 & 884021 & 933894 & 1183289 & 445745 & 320996 & 527804 & 266172 & 425046 & \\ 290507 & 1001799 & 926219 & 1016654 & 750816 & 146923 & 495992 & 280405 & & \\ 310608 & 1108250 & 776189 & 1562400 & 272482 & 352053 & 206286 & & & \\ 443160 & 693190 & 991983 & 769488 & 504851 & 470639 & & & & \\ 396132 & 937085 & 847498 & 805037 & 705960 & & & & & \\ 440832 & 847631 & 1131398 & 1063269 & & & & & & \\ 359480 & 1061648 & 1443370 & & & & & & & \\ 376686 & 986608 & & & & & & & & \\ 344014 & & & & & & & & \end{array}$

with exposure factors

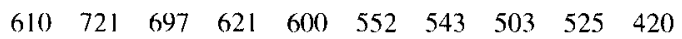


The exposures for each year of business are divided into the claims data before the analysis is carried out.

We now apply the model given by equation (11). This relates the row parameters recursively, and also allows the development parameters to evolve. Attention will be focussed on the development parameters, as it is the evolution of the run-off shape which is the subject of this paper.

In order to illustrate the effect of the model, the state variances have been chosen as follows.

$$
\operatorname{var}\left(e_{i j}\right)=0.116, \operatorname{var}\left(\alpha_{t} \mid \alpha_{i-1}\right)=0.0289, \operatorname{var}\left(\beta_{i j} \mid \beta_{i-1, j}\right)=0.01
$$

These values have been chosen in line with Verrall (1989). It should be emphasised that it is possible to estimate these from the data if that is appropriate. Also, they can be varied in order to gauge their effect. They do not have to be constant: sharp changes in the run-off shape can be modelled by putting a larger variance for the development factors at the appropriate point. Table 1 shows the estimates of $\mu$ and of the row parameters, $\alpha_{i}$. Also shown for comparison purposes are the estimates from the standard model, given by equation (2). Table 2 shows the estimates of the column parameters, $\beta_{i j}$, for columns 2 to 10 in rows 1 to 9 . The final row in this table shows the estimates from the standard model.

TABLE 1

\begin{tabular}{lcc}
\hline & Standard Model & State Space Model \\
\hline Overal Mean & 6.106 & 6.126 \\
Row Parameters & 0.194 & 0.184 \\
& 0.149 & 0.168 \\
& 0.153 & 0.194 \\
& 0.299 & 0.291 \\
& 0.412 & 0.387 \\
& 0.508 & 0.469 \\
& 0.673 & 0.534 \\
& 0.495 & 0.524 \\
& 0.602 & 0.536 \\
\hline
\end{tabular}

TABLE 2

\begin{tabular}{|c|c|c|c|c|c|c|c|c|c|}
\hline & 2 & 3 & 4 & 5 & 6 & 7 & 8 & 9 & 10 \\
\hline & \multicolumn{9}{|c|}{ State Space Model } \\
\hline 1 & 0.925 & 0.886 & 0.914 & 0.383 & 0.025 & -0.175 & -0.479 & -0.074 & \multirow[t]{9}{*}{-1.413} \\
\hline 2 & 0.918 & 0.895 & 0.945 & 0.361 & -0.0349 & -0.135 & -0.461 & -0.0628 & \\
\hline 3 & 0.920 & 0.907 & 0.964 & 0.361 & -0.0797 & -0.130 & -0.447 & & \\
\hline 4 & 0.918 & 0.920 & 0.980 & 0.332 & -0.050 & -0.161 & & & \\
\hline 5 & 0.895 & 0.942 & 0.951 & 0.352 & -0.0264 & & & & \\
\hline 6 & 0.894 & 0.960 & 0.940 & 0.375 & & & & & \\
\hline 7 & 0.890 & 0.990 & 0.944 & & & & & & \\
\hline 8 & 0.898 & 1.014 & & & & & & & \\
\hline 9 & 0.897 & & & & & & & & \\
\hline \multicolumn{10}{|c|}{ Standard Model } \\
\hline & 0.911 & 0.939 & 0.965 & 0.383 & -0.005 & -0.118 & -0.439 & -0.054 & -1.393 \\
\hline
\end{tabular}


The column parameters can be converted into the more familiar development factors, using equation (3). For the state space model, this is applied to each row separately. The results are shown in Table 3.

TABLE 3

\begin{tabular}{|c|c|c|c|c|c|c|c|c|c|}
\hline & 2 & 3 & 4 & 5 & 6 & 7 & 8 & 9 & 10 \\
\hline \multicolumn{10}{|c|}{ State Space Model } \\
\hline 1 & 3.522 & 1.688 & 1.419 & 1.174 & 1.104 & 1.077 & 1.053 & 1.075 & \multirow{9}{*}{1.018} \\
\hline 2 & 3.504 & 1.698 & 1.432 & 1.168 & 1.097 & 1.080 & 1.053 & 1.076 & \\
\hline 3 & 3.511 & 1.705 & 1.438 & 1.167 & 1.092 & 1.080 & 1.054 & & \\
\hline 4 & 3.505 & 1.716 & 1.443 & 1.161 & 1.094 & 1.077 & & & \\
\hline 5 & 3.447 & 1.744 & 1.431 & 1.165 & 1.097 & & & & \\
\hline 6 & 3.444 & 1.758 & 1.423 & 1.169 & & & & & \\
\hline 7 & 3.435 & 1.783 & 1.419 & & & & & & \\
\hline 8 & 3.454 & 1.799 & & & & & & & \\
\hline 9 & 3.452 & & & & & & & & \\
\hline \multicolumn{10}{|c|}{ Standard Model } \\
\hline & 3.488 & 1.733 & 1.434 & 1.169 & 1.098 & 1.080 & 1.054 & 1.075 & 1.018 \\
\hline
\end{tabular}

We will concentrate on the estimates of the parameters, and not show their standard errors (although these are available). It can be clearly seen that the development parameters have been allowed to evolve. The first parameter seems to be decreasing, while the second one is increasing. Patterns such as this can give useful insights into the changes in the run-off shape.

\section{CONCLUSIONS}

A state space model has been suggested which allows the development factors to evolve recursively. The model is not bound by the strong assumption made by the chain ladder technique that the run-off shape is the same for each accident year.

It may not be clear what parameter estimates should be used for forecasting the future development of the triangle. The most sensible estimates would be the latest ones. These are

$$
\begin{array}{lllllllll}
3.452 & 1.799 & 1.419 & 1.169 & 1.097 & 1.077 & 1.054 & 1.076 & 1.018
\end{array}
$$

compared with those of the ordinary chain ladder model:

$$
\begin{array}{lllllllll}
3.488 & 1.733 & 1.434 & 1.169 & 1.098 & 1.080 & 1.054 & 1.075 & 1.018
\end{array}
$$

The advantage of using the estimates from the dynamic model are that they are more likely to reflect the most recent run-off shape, which the best indication of future development. In particular, if large changes have occured in the development parameters, the straightforward estimates may be unreliable. The usual chain ladder technique does not weight the data according to the time since it was received. The first rows have the same effect on the estimates of the development parameters from 
this point of view as the more recent rows. The dynamic model gives more weight to recent data, by allowing the parameter to evolve.

It would also be straightforward to allow a sudden change in the run-off evolution by allowing the development factors to change suddenly. This can be done by using a suitably large variance for the stochastic disturbances.

\section{ACKNOWLEDGEMENT}

This research was performed under E.C. contract SPES-CT91-0063.

\section{REFERENCES}

Kremer, E. (1982) IBNR-Claims and the Two-Way Model of ANOVA. Scand. Act. J., Vol. 1, 47-55. Renshwa, A.E. (1989) Chain Ladder and Interactive Modelling (Claims Reserving and Glim). Journal of the Institute of Actuaries 1989, Vol, 116, Part. 111, 559-587.

TAYlor, G.C. and AsHe, F.R. (1983) Second Moments of Estimates of Outstanding Claims. $J$ of Econometrics, Vol. 23, 37-61.

Verrall R.J. (1989) A State Space Representation of the Chain Ladder Linear Model. Journal of the Institute of Actuaries 1989, Vol. 116, Part. 111, 589-610.

Verrall, R. J. (1990) Bayes and Empirical Bayes Estimation for the Chain Ladder Model. ASTIN Bulletin 1990, Vol. 20, No. 2, 217-243.

Verrall, R.J. (1991a) On the Unbiased Estimation of Reserves from Loglinear Models. Insurance: Mathematics and Economics 1991, Vol. 10, No. 1, 75-80.

VERRALL, R.J. (199lb) Chain Ladder and Maximum Likelihood. Journal of the Institute of Actuaries 199I, Vol. 118, Part. III, No. 471, 489-499.

\section{R. J. VerRaLl}

Department of Actuarial Science and Statistics,

City University, London. 